\title{
Desarrollo de la Imagen corporal, la autoestima y el autoconcepto físico al finalizar la Educación Primaria*
}

\section{Development of Body Image, Self-esteem and Physical self-Concept at the end of Primary Education}

Recepción: 01 Marzo 2016 | Aprobación: 27 Enero 2017

\author{
Juan Palomares Cuadros ${ }^{\mathrm{a}}$ \\ Universidad de Granada, España \\ ORCID: http://orcid.org/0000-0003-0777-9670 \\ Jóse Manuel Cuesta Santos \\ Universidad de Granada, España \\ Manuel Estévez Díaz \\ Universidad de Granada, España \\ Beatriz Torres Campos \\ Universidad de Granada, España
}

a Autor de correspondencia. Correo electrónico: jpcuadros@andaluciajunta.es

Para citar este artículo: Palomares, J., Cuesta, J., Estévez, M., \& Torres, B.(2017). Desarrollo de la Imagen corporal, la autoestima y el autoconcepto físico al finalizar la Educación Primaria. Universitas Psychologica,16(4), 1-13. https://doi.org/10.11144/Jav eriana.upsy16-4.dica

\section{RESUMEN}

En este trabajo se analiza la relación entre la satisfacción con la imagen corporal, la autoestima y el autoconcepto físico. Los participantes fueron 310 alumnos, de 50 y $6^{0}$ de Educación Primaria, y 22 profesores en activo, de la zona educativa Motril-Costa (España). Como técnicas cuantitativas, se utilizaron: el Cuestionario de Siluetas Corporales, Cuestionario de Autoconcepto Físico y la Escala de Autoestima de Rosenberg. Como técnica cualitativa, se utilizó la entrevista semiestrucutrada. Los resultados muestran que difiere la imagen que tienen y que les gustaría tener. También se aprecia que los chicos tienen un mayor nivel de autoestima. Son las chicas las que se consideran menos hábiles para realizar actividad física y disfrutan en menor medida de estas actividades.

Palabras clave

preadolescencia; imagen corporal; autoconceptofísic; autoestima.

\begin{abstract}
In this paperis analyzed the relationshipbetween satisfaction withbody image,self-esteem andphysical self.Participants were310students,grades 5 and 6primary and22practicing teachers, the educationalareaMotrilCosta (Spain). Asquantitative techniquesQuestionnairebody silhouettes, Physical SelfQuestionnaire and theRosenbergSelf-Esteem Scale.Asthe qualitative technique was used semi-structured interview. The results show thatdifferent fromthe image they haveand would like tohave.It is also appreciatedthat the boyshave a higher levelofself-esteem.It isthe girls whoare consideredless abletoenjoyphysical activityandto a lesser extentof these activities.

Keywords

preadolescence; body image; self-esteem; physical self-concept.
\end{abstract}

| Universitas Psychologica | Colombia | V. I6 | No.4 | 2017 | ISSN i657-9267 | 
La investigación sobre los factores que intervienen en el proceso de enseñanza y aprendizaje, es crucial para que el alumnado reciba una educación integral. Entre los factores que influyen en este proceso, durante el periodo de preadolescencia y adolescencia, son claves las relaciones que el alumnado desarrolla con su cuerpo, ya que estas van a influir en su autoestima, en la perspectiva sobre su imagen corporal y en las influencias que sobre ello tiene el grupo de iguales (Schmidt, Valkanover, Roebers, \& Conzelmann, 2013). Además, también existen evidencias científicas sobre la relación bidireccional entre autoconcepto y rendimiento escolar (Marsh \& O'Mara, 2008; Bryk, Harding, \& Greenberg, 2012) o del bienestar del estudiante en la escuela (Standage, Gillison, Ntoumanis, \& Treasure, 2012).

Con el fin de centrarnos en este periodo del desarrollo humano, es decir en la preadolescencia, hay que señalar que como indican Morgan, Saunders y Lubans (2012), esta etapa tiene más que ver con lo social que con lo físico. El alumnado tiene poco poder de decisión, en cambio es consumista; y eso le lleva a que determinados gustos e intereses se incentiven por una conveniencia del mercado. También, suelen manifestar afinidad hacia una determinada estética impuesta por la sociedad contemporánea, esto hace que los más jóvenes presenten una preocupación excesiva por su cuerpo y lleven a cabo conductas de riesgo que pueden producir trastornos de la conducta alimentaria, como anorexia y bulimia nerviosa (Baile, Raich, \& Garrido, 2003; Thompson, 2004, Xu et al., 2010), y además, hayan transcendido al mundo de la salud, tanto física, como mental (Kriemler et al., 2011); y en la adolescencia se haya convertido en un problema de salud pública (Käll, Nilsson, \&Lindén, 2014).

De la misma manera, de los diez factores de riesgo identificados por la OMS como claves para el desarrollo de las enfermedades crónicas, cinco están estrechamente relacionados con la alimentación y el ejercicio físico (OMS, 2014). La prevalencia de la obesidad en la infancia y la adolescencia ha ido aumentando de manera alarmante durante los últimos treinta años en los países desarrollados (Dietz \& Robison, 2005) e incluso, de forma incipiente, en los países considerados "en vías de desarrollo" (Onís \& Blössner, 2000). La alimentación poco saludable y la falta de práctica de actividad física con regularidad, son causas importantes de algunas de las patologías crónicas de mayor incidencia, y ambas son susceptibles de modificarse.

A ello hay que añadirle, desde una perspectiva sociocultural, al menos en las sociedades occidentales, los modelos de belleza ideal que han girado principalmente en torno a la delgadez femenina y el desarrollo muscular masculino (Grogan,1999).

Los profesionales de la educación física utilizan a menudo la percepción de la imagen corporal como medida de la autoestima y del grado de satisfacción con la propia imagen (Thompson \& Gray, 1995; Dowson \& Hhendersen, 2001). Sin duda, múltiples factores biológicos, sociales y culturales modulan esta percepción, pero en algunos casos, estos factores son difíciles de valorar (Espinoza, Rodríguez, Gálvez, Vargas, \& Yáñez, 2011).

Ante esta realidad social, docentes y académicos, como por ejemplo Fuentes (2011), señalan que los educadores y profesionales de la educación física, no deben permanecer indiferentes, ya que esta área es un medio muy valioso para educar, formar y fomentar actitudes y valores positivos, e incidir además en la creación, el mantenimiento y/o mejora de hábitos de salud, a través de la práctica de actividad física, que puedan contribuir a una vida de calidad en el ámbito personal, social y ambiental de los niños y niñas en edad escolar. Debido a estas razones, el equipo de trabajo docente de la zona educativa Motril-Costa (Consejería de Educación de la Junta de Andalucía [CEJA], 2012) planteó realizar un estudio de la situación del alumnado, con el fin de realizar una concreción curricular zonal, acorde con la realidad del alumnado preadolescente.

En consecuencia, en este estudio se pretende conocer la realidad, dentro de su contexto socio cultural, de la relación entre la satisfacción con la imagen corporal, la autoestima y el autoconcepto 
físico, del alumnado preadolescente de la zona educativa Motril-Costa de la provincia de Granada, expresada por el alumnado y percibida por el profesorado, con el fin de mejorar la calidad educativa.

\section{Diseño y metodología de la investigación}

\section{Diseño metodológico}

Se realizó una investigación descriptiva e interpretativa, a través de una integración metodológica de los datos obtenidos (Johnson \& Christensen, 2014), que fueron complementados de manera cuantitativa con las indagaciones obtenidas del análisis del discurso del profesorado de educación física, tanto de colegios públicos como privados concertados (Latorre, Rincón, \& Arnal, 2003).

Esta investigación se dividió en dos fases, en la primera fase (enero-junio de 2012), se realizó el diligenciamiento de los cuestionarios, para la obtención de información de la satisfacción con la imagen corporal, con la autoestima y con el autoconcepto físico, así como su percepción de la educación física escolar:

1. Cuestionario sociodemográfico.

2. Cuestionario de Siluetas Corporales (Collins, 1991), en el que se analiza la satisfacción con la Imagen corporal a través de las variables: silueta percibida y silueta deseada.

3. Cuestionario de Autoconcepto Físico (CAF; Goñi, Ruiz de Azúa, \& Rodríguez, 2006), en el que se analiza la percepción de la torpeza motriz, la aversión hacia el ejercicio físico, la percepción del rostro, la figura, la musculatura y la apariencia física.

4. Escala de Autoestima de Rosenberg (1965), para analizar el sentimiento de orgullo hacia uno mismo y satisfacción consigo mismo.

Una vez recopilados todos los datos, se realizó un análisis descriptivo, y para establecer la relación que existe entre la satisfacción con la imagen corporal y la autoestima, se trabajó con la correlación entre los datos obtenidos de la silueta percibida como propia y la silueta deseada, a través de las correlaciones de Spearman y de Pearson.

En la segunda fase (entre septiembre a febrero de 2013), se elaboró y se implementó un protocolo de entrevista semi-estructurada, a través de los expertos de grupo de investigación HUM-727 de la Universidad de Granada y las consideraciones expresadas por Taylor y Bogdan (1987); Miles y Huberman (1994) y Berenguera et al. (2014).

Dicho protocolo estuvo fundamentalmente orientado a la indagación sobre la visión que sobre el objeto de estudio tenía el profesorado. El proceso estuvo en todo momento orientado al descubrimiento, donde a menudo las entrevistas comenzaban a modo de relato biográfico. Del mismo modo, los datos fueron en todo momento complementados con las sucesivas conversaciones informales mantenidas. Dicho proceso de complementariedad y triangulación de información se hizo extensible a seis informantes clave (de entre el profesorado de los centros) que fueron consultados de forma habitual a lo largo de todo el trabajo de campo. Se llevó a cabo un análisis de contenido. Tras la primera lectura general del material transcrito, se identificaron los temas emergentes, y se establecieron las dimensiones y subdimensiones de análisis. La clasificación conceptual de los códigos y las categorías se construyeron siguiendo procedimientos tanto deductivo como inductivos. El proceso se inició con una lista predeterminada de temas a ser explorados, en relación con los análisis estadísticos. Sin embargo, cualquier categoría o subcategoría emergente durante el proceso de examen de los resultados fue incorporada al análisis. Todo el proceso de generación de conceptos y categorías, así como el proceso de codificación fue realizado por el investigador principal en colaboración otro miembro del equipo. Este procedimiento no solo fue parte del proceso de triangulación, sino también, una forma de añadir una mayor confiabilidad al análisis. 


\section{La Muestra}

Para el desarrollo de los aspectos cuantitativos de la investigación, los participantes fueron 310 alumnos, de $5^{\mathrm{o}}$ y $6^{\mathrm{o}}$ de Educación Primaria, en donde el $48.38 \%$ fueron chicos $(\mathrm{n}=150)$, $y$ el $51.62 \%$ chicas $(\mathrm{n}=160)$, con edades comprendidas entre los 10 y 12 años con una media de edad de 11.23 en las chicas, y 10.99 en los chicos. La muestra se obtuvo mediante proceso aleatorio por conglomerados, polietápico estratificado (Ramos, Catena, \& Trujillo, 2004). El error para el conjunto de la muestra, utilizando la fórmula de poblaciones finitas y asumiendo la hipótesis de máxima varianza poblacional $(\mathrm{p}=\mathrm{q}$ $=50$ ) es de $4.25 \%$ al nivel de confianza del $95 \%$ (Arkin \& Colton, 1984).

En cuanto a la indagación y profundización en aquellos aspectos de interés para el objetivo general de la investigación, que surgieron del análisis cuantitativo, utilizamos el denominado "muestreo secuencial conceptualmente conducido" (Hammersley \& Atkinson, 1995), con profesorado que participaba en el proceso de enseñanza y aprendizaje del alumnado. Se realizaron 22 entrevistas semi-estrucuturadas.

Los criterios seguidos para seleccionar a los individuos fueron:

Abarcar con la muestra todas las áreas de la zona educativa (CEJA 2012).

Mantener en la muestra una proporción similar a la que existe en la población total en cuanto al tipo de centro: privado-concertado y público.

Tener posibilidad de acceder a la muestra.

Que los entrevistados fueran representativos del profesorado de educación física de la zona.

\section{Consideraciones éticas}

Elestudio fue aprobado porel Comité de Éticade la Universidad de Granada, cuya aceptación implica asumir las normas éticas internacionales APA y la Declaración de Helsinki. Se pidió un consentimiento informado, por escrito, a todos los centros educativos y al profesorado participante, informando a toda la comunidad educativa implicada en la investigación, sobre el objeto de estudio, el modo de proceder, el uso que se haría de la misma, y su importancia social.

\section{Resultados}

Se presentan los principales resultados en dos apartados: en el primero, la relación entre la satisfacción con la imagen corporal y la autoestima; y en el segundo, la relación entre la satisfacción con la imagen corporal y el autoconcepto físico. En ambos apartados, se completan las evidencias cuantitativas con los aspectos cualitativos que tratan de profundizar y dar una explicación contextualizada a las causas de estas evidencias, desde la óptica del profesorado, aportando algunas citas textuales, las cuales llevan los códigos de sus análisis incorporados.

Relación entre la satisfacción con la imagen corporal y la autoestima

\section{Gráfico 1}

Silueta elegida como propia por el total de la muestra

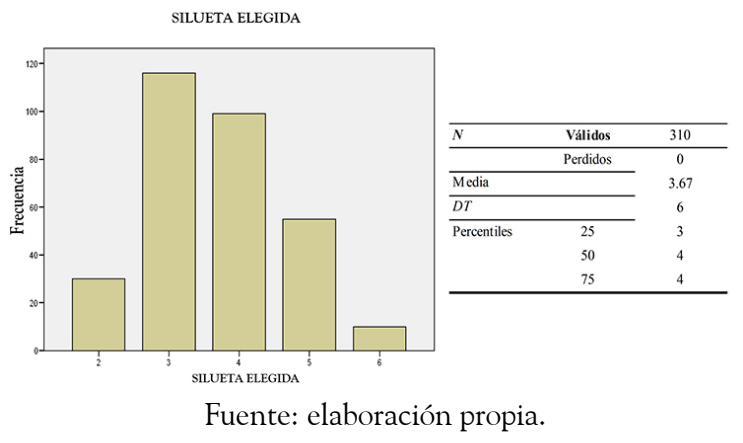


Gráfico 2

Silueta deseada por el total de la muestra SILUETA ELEGIDA

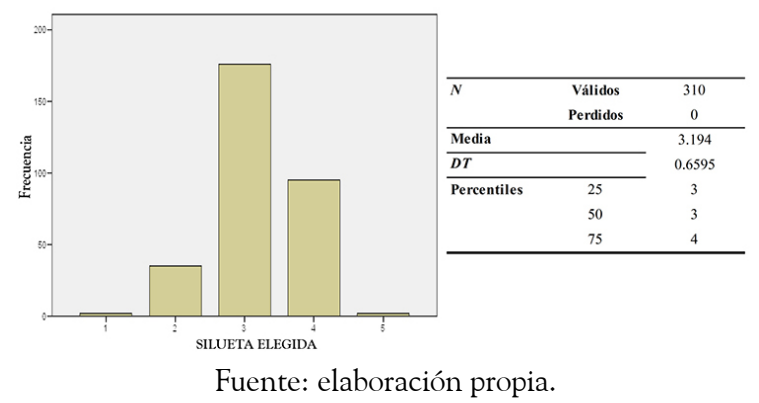

TABLA 1

Comparación de la muestra global en la selección de la silueta percibida y la silueta deseada

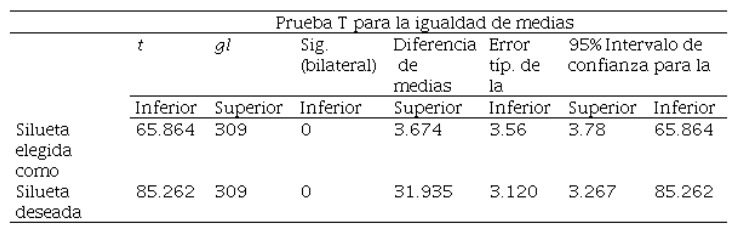

Fuente: elaboración propia.

\section{TABLA 2}

Correlaciones de Pearson entre la silueta percibida y la silueta deseada

\begin{tabular}{llll}
\hline Correlación de Pearson & $\begin{array}{l}\text { Silueta } \\
\text { propia }\end{array}$ & $\begin{array}{l}\text { Silueta } \\
\text { deseada }\end{array}$ \\
\hline \multirow{2}{*}{$\begin{array}{l}\text { Correlación } \\
\text { de Pearson }\end{array}$} & 1 & $0.367\left(^{* *}\right)$ \\
propia & $\begin{array}{l}\text { Sig. (bilateral) } \\
\end{array}$ & $\mathrm{N}$ & 0 \\
\hline & $\begin{array}{l}\text { Correlación } \\
\text { de Pearson }\end{array}$ & $0.367\left(^{* *}\right)$ & 1 \\
$\begin{array}{l}\text { Silueta } \\
\text { deseada }\end{array}$ & Sig. (bilateral) & 0 & \\
& $\mathrm{~N}$ & 310 & 310 \\
\hline
\end{tabular}

Fuente: elaboración propia.
TABLA 3

Correlaciones de Spearman entre la silueta percibida y la silueta deseada

\begin{tabular}{llll}
\hline Rho de Spearman & $\begin{array}{l}\text { Silueta } \\
\text { propia }\end{array}$ & $\begin{array}{l}\text { Silueta } \\
\text { deseada }\end{array}$ \\
\hline \multirow{4}{*}{$\begin{array}{l}\text { Correlación de } \\
\text { correlación } \\
\text { propia }\end{array}$} & 1 & $0.374\left(^{* *}\right)$ \\
& Sig. (bilateral) & $\mathrm{N}$ & 0 \\
& $\begin{array}{l}\text { Correlación de } \\
\text { Correlación } \\
\text { Silueta } \\
\text { deseada }\end{array}$ & $0.374\left(^{* *}\right)$ & 1 \\
\hline & $\mathrm{N}$ & 0 & \\
\hline
\end{tabular}

Fuente: elaboración propia.

En la relación que existe entre la satisfacción con la imagen corporal y la autoestima, la correlación de Spearman, entre los datos obtenidos de la silueta percibida y la deseada, indicaba un coeficiente de correlación positivo $0.376(* *)$. También se halló una significación bilateral de $(\mathrm{p}=0)$, y la correlación de Pearson arrojaba un valor de $0.385(* *)$, y una significación bilateral de $(\mathrm{p}=0)$. Estos resultados llevan a considerar un grado de insatisfacción corporal, que mustra que la imagen con la que el alumnado se percibe, difiere de la imagen que le gustaría tener.

TABLA 4

Ítem II.1: Tengo buena figura para mi edad

\begin{tabular}{|c|c|c|c|c|c|c|c|c|c|c|c|c|}
\hline & \multicolumn{8}{|c|}{ Curso/Sexo } & \multirow{2}{*}{\multicolumn{4}{|c|}{ Totales por sexo }} \\
\hline & \multicolumn{4}{|c|}{$5^{\circ}$ Curso } & \multicolumn{4}{|c|}{$6^{\circ}$ Curso } & & & & \\
\hline & Chi & $\cos$ & Chi & cas & Chi & $\cos$ & Chi & cas & \multicolumn{4}{|c|}{ Chicos Chicas } \\
\hline & Frec & $\%$ & Frec & $\%$ & Frec & $\%$ & Frec & $\%$ & Frec & $\%$ & Frec & $\%$ \\
\hline $\begin{array}{l}\text { Min } \\
1\end{array}$ & 5 & 6.3 & 3 & 3.3 & 1 & 1.4 & 2 & 2.9 & 6 & 4 & 5 & 3.1 \\
\hline 2 & 4 & 5.1 & 4 & 4.4 & 4 & 5.6 & 4 & 5.8 & 8 & 5.3 & 8 & 5 \\
\hline 3 & 7 & 8.9 & 4 & 4.4 & 2 & 2.8 & 7 & 10.1 & 9 & 6 & 11 & 6.9 \\
\hline 4 & 23 & 29.1 & 20 & 22 & 14 & 19.7 & 11 & 15.9 & 37 & 24.7 & 31 & 19.4 \\
\hline 5 & 20 & 25.3 & 19 & 20.9 & 19 & 26.8 & 23 & 33.3 & 39 & 26 & 42 & 26.3 \\
\hline $\begin{array}{l}\text { Máx } \\
6\end{array}$ & 20 & 25.3 & 41 & 45.1 & 31 & 43.7 & 22 & 31.9 & 51 & 34 & 63 & 39.4 \\
\hline Total & 79 & 100 & 91 & 100 & 71 & 100 & 69 & 100 & 150 & 100 & 160 & 100 \\
\hline
\end{tabular}

Fuente: elaboración propia. 
TABLA 5

Ítem II.17: Tengo poca musculatura

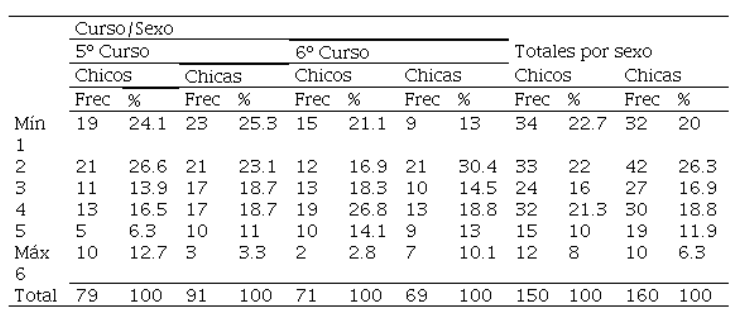

Fuente: elaboración propia.

También aparecieron diferencias significativas cuando se comparaba el Ítem II.1: Tengo buena figura para mi edad, con el ítem II.17: Tengo poca musculatura (test de Chi-cuadrado: $\mathrm{p}=0.034$ ) en el caso de los chicos, lo que se interpreta como que aquellos alumnos que manifiestan tener una buena figura para su edad, consideran tener una musculatura adecuada.

Cuando se realizaba la correlación de Spearman entre los resultados obtenidos en la percepción de la Silueta percibida como propia, y se comparaban con los datos encontrados en el Ítem II.1: Tengo buena figura para mi edad, resultaba una correlación negativa $-0.347(* *)$, y unas diferencias estadísticamente significativas de $(\mathrm{p}=0)$. De la misma manera, en la correlación de Pearson obtenía un valor de $-0.358(* *)$, y bilateral de $(\mathrm{p}=0)$, lo que supone que aquellos alumnos y alumnas que se perciben con una silueta inadecuada para su tipología, son aquellos que indican que no tienen una figura adecuada para su edad.

Este significativo grado de insatisfacción corporal es un lugar común entre el profesorado, que plantea la falta de valores y principios educativos explícitos en los proyectos educativos de sus centros, encaminados a mejorar esta situación y contribuir a una mejora de la educación integral:

En el día a día aparecen muchos problemas derivados de su insatisfacción corporal. Cuando hay cierta confianza, te preguntan sobre este tema, se observa a las chicas más preocupadas. Creo que es cuando hay que empezar a atajar, es decir, a prevenir sobre este asunto. Sería muy eficaz la coordinación con las familias y los equipos de orientación. Profesor 4 (pág. 278-288).

Otro dato significativo aparece al diferenciar por grupos, correlacionando las variables silueta percibida como propia y los datos encontrados en el Ítem II.1: Tengo buena figura para mi edad. Se comprobó que son las chicas las que muestran una correlación negativa mayor $-0.374(* *)$, frente a $-0.339(* *)$ de los chicos. En ambos casos a nivel bilateral aparecen diferencias significativas, con un valor de $(\mathrm{p}=0)$.

El profesorado argumenta dos causas relacionadas entre sí, como posible justificación de este hecho. Por un lado, la influencia de los medios de comunicación en la figura ideal que debemos tener, y por otra, la influencia del medio ambiente, donde el clima subtropical que caracteriza a la zona, hace que el cuerpo este "menos escondido", como señalaba un informante, lo que causa que el alumnado preste mayor atención al físico y a su vez, se encuentre más condicionado por esta imagen.

TABLA 6

Ítem II.3. Tengo buena apariencia física

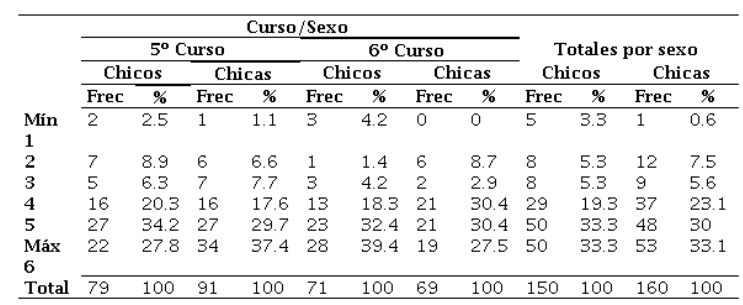

Fuente: elaboración propia.

Gráfico 3

Ítem III.32.- Siento que no tengo demasiadas cosas de las que sentirme orgulloso
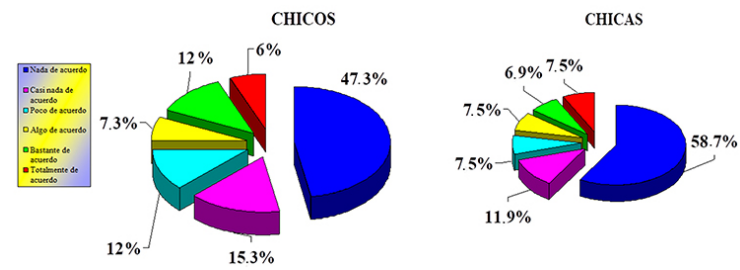

Fuente: elaboración propia

También, como se aprecia al comprobar la relación entre el ítem II.3: Tengo buena apariencia física, y el ítem III.32: Siento que no 
tengo demasiadas cosas de las que sentirme orgulloso/ $a$, en los resultados del test de Chi cuadrado. En los resultados del test de Chi cuadrado aparecieron diferencias significativas al hacer la comparativa por sexo. Así en el grupo de chicas el valor de $(\mathrm{p}=0.002)$, al contrario que los chicos, lo que evidencia que las chicas están más preocupadas por su apariencia física que los chicos. De la misma manera un profesor los indicaba así:

Las chicas a estas edades suelen sufrir cambios corporales más evidentes. Dichos cambios y los estereotipos socioculturales las hacen más sensibles al tema en cuestión. Habría que buscar la manera de formarnos mejor en este tema, ya que es más de sicología en el que no estoy tan puesto. Profesor 11 (pág 1273-1274) MGF

También, el profesorado considera que, aunque las diferencias de autoestima no son muy altas a nivel de sexo, sí observan que los chicos tienen un mayor nivel que las chicas. Del mismo modo que empiezan a aceptar su cuerpo en general, pero es el momento en que en algunos casos empiezan los problemas relacionados con su imagen corporal y su autoestima. Reclaman una relación más estrecha y abierta entre equipos docentes, familias, sicólogos y expertos en salud, con el fin de abordar este tema desde una óptica más amplia, ya que confirman que es crucial para una transición positiva a la educación secundaria.

Relación entre la satisfacción con la imagen corporal y el Autoconcepto físico

\section{TABLA 7}

Ítem II.7.Soy torpe para hacer actividad física

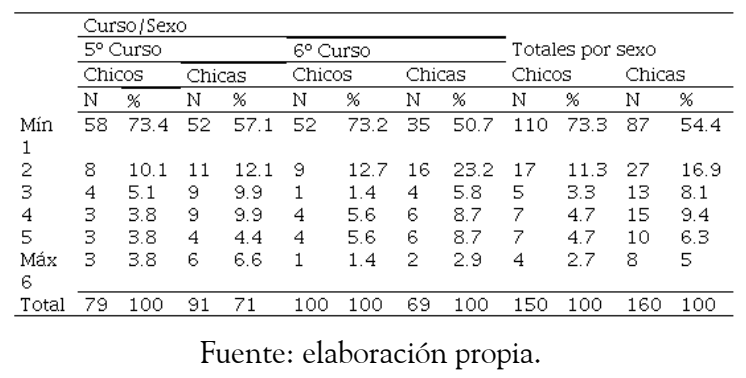

\section{Gráfico 4}

Ítem III.28. En general, estoy satisfecho/a conmigo mismo/a

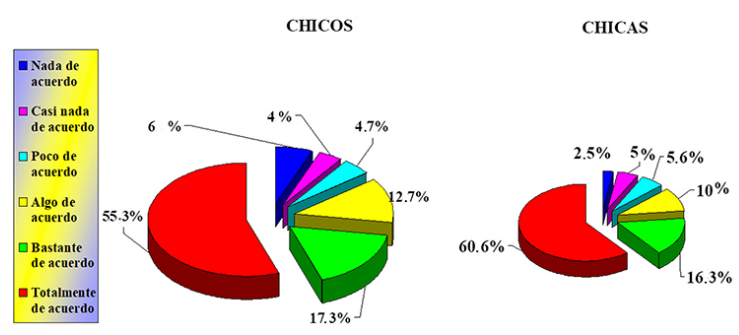

Fuente: elaboración propia.

Al tratar de verificar la presencia o ausencia de insatisfacción corporal provocada por su percepción sobre su grado de habilidad o torpeza motriz, hemos procedido a comparar las respuestas obtenidas en el Ítem II.7:Soy torpe para hacer actividad física, y el Ítem III.28: En general, estoy satisfecho conmigo mismo, en donde se encontraron diferencias ligeramente significativas en el test de Chi-cuadrado ( $\mathrm{p}=$ 0.006), es decir, el alumnado que se considera torpe motrizmente, se encuentra insatisfecho consigo mismo y viceversa.

\section{Gráfico 5}

Item II.7: Soy torpe para hacer actividad física.

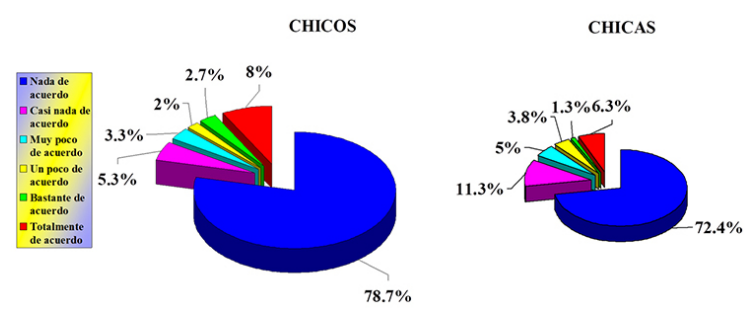

Fuente: elaboración propia.

Al profundizar en la torpeza o habilidad motriz del alumnado, encontramos que al comparar el ítem II.7: Soy torpe para hacer actividad física, y el ítem II.8: Detesto las cosas relacionadas con el ejercicio físico, encontramos que, tanto en chicos como en chicas, se encuentran diferencias significativas en el test de Chi-cuadrado, con un valor de $(\mathrm{p}=0.00)$. Al realizar la correlación de Pearson entre estos dos ítems, las chicas arrojaron un valor de $0.426\left(^{* *}\right)$, frente a los chicos, con un valor de $0.190(* *)$. En la correlación de Spearman, para el sexo femenino, existe una 
correlación positiva de $(0.463(* *)$, frente a los chicos que obtienen un valor de $0.295(* *)$, quienes presentan mayor correlación, lo que evidencia que en mayor medida son las chicas, las que se consideran más torpes para realizar actividad física y detestan en mayor grado estas actividades.

La percepción del profesorado de educación física coincide plenamente, pues infiere un déficit de actividad física y desarrollo motriz, lastrado durante la educación primaria, que influye en su nivel de autoconcepto, en donde se aprecia que el alumnado que infravalora sus habilidades motrices, tiene menos motivación en el aula, lo que es más frecuente en las chicas.

Según el profesorado, los chicos manifiestan de manera ligeramente superior tener un mejor autoconcepto físico que las chicas.Los chicos se perciben con más capacidad que las chicas, aunque ambos grupos se consideran con la suficiente capacidad para realizar los ejercicios físicos. Del mismo modo, ambos grupos creen poseer resistencia física, pero los chicos se muestran más seguros, y con menos posibilidades de cansarse que las chicas cuando realizan ejercicio físico. Por lo anterior, el profesorado cree que una intervención consciente y programada desde el primer ciclo de primaria hasta el segundo ciclo de secundaria, mejoraría la autoestima y el autoconcepto físico, y ofrecería un repertorio más amplio de juegos y pre-deportes que discriminasen positivamente a las chicas.

\section{TABLA 8}

Ítem II.4. Tengo un rostro agradable

\begin{tabular}{|c|c|c|c|c|c|c|c|c|c|c|c|c|}
\hline & \multicolumn{8}{|c|}{ Curso/Sexo } & \multirow{2}{*}{\multicolumn{4}{|c|}{ Totales por sexo }} \\
\hline & \multicolumn{4}{|c|}{$5^{\circ}$ Curso } & \multicolumn{4}{|c|}{$6^{\circ}$ Curso } & & & & \\
\hline & \multicolumn{2}{|c|}{ Chicos } & \multicolumn{2}{|c|}{ Chicas } & \multicolumn{2}{|c|}{ Chicos } & \multicolumn{2}{|c|}{ Chicas } & \multicolumn{2}{|c|}{ Chicos } & \multicolumn{2}{|c|}{ Chicas } \\
\hline & $\mathrm{N}$ & $\%$ & $\mathrm{~N}$ & $\%$ & $\mathrm{~N}$ & $\%$ & $\mathrm{~N}$ & $\%$ & $\mathrm{~N}$ & $\%$ & $\mathrm{~N}$ & $\%$ \\
\hline $\begin{array}{l}\text { Mín } \\
1\end{array}$ & 1 & 1.3 & 2 & 2.2 & 2 & 2.8 & 2 & 2.9 & 3 & 2 & 4 & 2.5 \\
\hline 2 & 4 & 5.1 & 2 & 2.2 & 1 & 1.4 & 2 & 2.9 & 5 & 3.3 & 4 & 2.5 \\
\hline 3 & 7 & 8.9 & 6 & 6.6 & 5 & 7 & 7 & 10.1 & 12 & 8 & 13 & 8.1 \\
\hline 4 & 21 & 26.6 & 12 & 13.2 & 13 & 18.3 & 14 & 20.3 & 34 & 22.7 & 26 & 16.3 \\
\hline 5 & 24 & 30.4 & 31 & 34.1 & 22 & 31 & 18 & 26.1 & 46 & 30.7 & 49 & 30.6 \\
\hline Máx & 22 & 27.8 & 38 & 41.8 & 28 & 39.4 & 26 & 37.7 & 50 & 33.3 & 64 & 40 \\
\hline Total & 79 & 100 & 91 & 100 & 71 & 100 & 69 & 100 & 150 & 100 & 160 & 100 \\
\hline
\end{tabular}

Fuente: elaboración propia.

Sobre el atractivo físico, en el análisis de los datos del ítem II.4: Tengo un rostro agradable, con el ítem II.3: Tengo buen apariencia física, encontramos en el test de Chi-cuadrado, que en ambos sexos aparecen diferencias significativas $(\mathrm{p}=0.00)$, al igual que al comparar el ítem II.3: Tengo buena apariencia física, con el ítem II.17: Tengo poca musculatura. De la misma manera, la relación entre la fuerza corporal y la musculatura se hace evidente también al comparar el ítem II.16: Soy una persona físicamente fuerte, y el ítem II.17: Tengo poca musculatura, en donde encontramos diferencias significativas en test de Chi-cuadrado en el grupo de chicos con un valor de $(\mathrm{p}=0)$, pero no en el grupo de chicas. Así, se reitera que los chicos consideran la musculatura como un elemento clave de su imagen corporal y, además, tienden a resaltar la fuerza como una cualidad importante en la práctica deportiva. De igual manera, el profesorado percibe que también lo consideran importante en su ámbito de acción social, donde se percibe una mayor aceptación social de aquellos que destacan por su físico y habilidades motrices. También argumentaron que en los últimos años, habían proliferado los centros de estética y deporte en los principales núcleos urbanos de la zona, y observaron las influencias de esta cultura corporal. El profesorado aboga por la necesidad de incorporar estrategias didácticas cooperativas, desarrollar una actitud crítica hacia la estética corporal, así como establecer una acción coordinada con el desarrollo de las actividades deportivas extraescolares.

\section{Discusión}

Después de examinar, en preadolescentes y en su profesorado, la relación entre la satisfacción con la imagen corporal, la autoestima y el autoconcepto físico, los resultados de este estudio muestran que tanto la imagen corporal, como la autoestima, son dos constructos multidimensionales que tienen implicación directa sobre el individuo y su entorno sociocultural (Rees, Caird, Dickson, Vigurs, \& Thomas, 2013).

En cuanto a la relación entre la imagen corporal y la autoestima, de los resultados obtenidos sobre su figura, el alumnado que se 
percibe con una silueta inadecuada, también muestra un grado significativo de insatisfacción corporal. Davison y McCabe (2006) encuentran que el alumnado insatisfecho con su imagen corporal, es aquel que también tiene menor grado de autoestima. Nuestro profesorado expresó la disconformidad existente en el alumnado, y matizó la mayor sensibilidad en las chicas, lo que supone un aspecto crucial a tener en cuenta a la hora de programar las sesiones de clase. En esta misma tendencia Dunn, Lewis y Patrick (2010) afirman que en la mayoría de los estudios con preadolescentes, a más del 50\% les gustaría ser más delgados.

Esta investigación contribuye a tratar de dar una explicación desde algunas de las áreas que Berk (2007) señala dentro de la autoestima: el autoconcepto físico y la imagen corporal. En los chicos, el análisis comparativo entre la silueta percibida y la silueta deseada, nos lleva a considerar un significativo grado de insatisfacción corporal, que encuentra el valor social de la musculatura como una causa de esta insatisfacción. En este sentido, tanto los datos del alumnado como las percepciones del profesorado coinciden con la reflexión presentada por Xu et al. (2010), cuando explican que el deseo de incremento de masa muscular entre los jóvenes chinos, se debe al incremento de la influencia de la cultura occidental diseminada a través de los medios, así como otros factores como la dieta, el socio-económico y los cambios en el rol del género. Estos datos también coinciden con los encontrados por Mellor et al. (2014), quienes concluyen que los chicos crecen, hoy en día, con insistente bombardeo de imágenes relacionadas con la apariencia física y la representación del hombre objeto, influyendo en su insatisfacción con su imagen corporal.

De la misma manera, el profesorado considera que, aunque las diferencias de autoestima no son muy altas a nivel de sexo, sí observan que los chicos de sus centros tienen un mayor nivel que las chicas, por lo que plantean para una educación de calidad, acciones educativas coordinadas con familias, equipos docentes y orientadores, tanto de primaria como de secundaria. Los entrevistados también coinciden con la opinión de Baldwin y Hoffmann (2002), que consideran que la autoestima no es un rasgo estático ni estable en el tiempo, sino más bien un índice dinámico y sujeto a cambios, que se ve influido por las experiencias a las que las personas nos vemos expuestas. Teniendo en cuenta que la adolescencia es un periodo en el que chicas y chicos deberán hacer frente a importantes cambios y resolver distintas tareas evolutivas, es de esperar que su nivel de autoestima experimente cambios y fluctuaciones, y como señala el profesorado encuestado, es a partir de estas edades cuando se observa preocupación por su imagen corporal, que influye en su nivel de autoestima. La obtención de una valoración positiva de sí mismo, que opera de forma automática e inconsciente, permite un desarrollo psicológico sano, en armonía con su medio circundante y, en especial, en su relación con los demás (Mellor et al., 2014).

En la relación entre la satisfacción con la imagen corporal y el autoconcepto físico, aparece que las chicas y chicos se encuentran a nivel evolutivo en el final de la tercera infancia y la preadolescencia, por lo que los cambios psico-somáticos aún no se han generalizado, y ello podría ser una de las razones para que se aprecien pocas diferencias significativas por sexo en los niveles de autoestima (Rees et al., 2013). Aunque el profesorado sí percibe diferencias y detecta problemas a medida que van surgiendo. Además, consideran que la falta de competencia motriz invita a que algunos escolares sean ridiculizados por sus compañeros y a ser excluidos de los juegos deportivos. Este fracaso incrementa los sentimientos de incompetencia, así como unos niveles mayores de ansiedad. Esta investigación aporta, en este sentido, que el alumnado que se considera torpe motrizmente, es el más insatisfecho, y al contrario aquellos que se consideran más hábiles, muestran mejor conformidad consigo mismos, como también ponen de manifiesto los estudios de Schmidt et al. (2013). Además, se puede confirmar, que en nuestra población, son más las chicas que los chicos, las que se consideran más torpes para realizar actividad física y las que también detestan estas actividades. 
Para el profesorado, la torpeza motriz es una causa para evitar la práctica de actividad física y dejar de disfrutar de un estilo de vida activo y saludable. Existe una responsabilidad entre los educadores y las instituciones educativas de atender estas necesidades como se atienden otro tipo de dificultades (Marsh \& Martin, 2011). Además, como señala Cumming et al. (2011), un autoconcepto físico positivo entre las adolescentes está asociado con un mayor grado de participación en la clase de educación física y en actividades físico-deportivas extraescolares.

Sobre el atractivo físico, estos datos están en consonancia con la mayoría de las investigaciones, que encuentran a las chicas más preocupadas por su aspecto físico, lo que incide en una mayor exigencia y menor satisfacción corporal, lo que ocurre en una amplia franja de edad (Klomsten, Skaalvik, \&Espnes, 2004; Infante, Goñi, \& Villarroel, 2011; Liu, Wu, \& Ming, 2015). Asimismo, como señalábamos anteriormente, los chicos consideran la musculatura como un elemento clave de su imagen corporal, y tienden a resaltar la fuerza como una cualidad importante en la práctica deportiva.

En este sentido, del análisis cualitativo emergió la relación entre la preocupación por la estética física y el reciente aumento de centros de belleza y deporte en la zona, que influyen en la actitud del alumnado. También surgió la necesidad de incorporar una intervención en valores, que contrarrestara de una manera positiva estas influencias, que a juicio de los entrevistados, rompen la adquisición de contenidos actitudinales, dado que el alumnado se encuentra expuesto durante más tiempo y sin haber desarrollado una competencia crítica suficiente, para poder reflexionar sobre ello.

En conclusión, existen evidencias científicas que certifican que niveles elevados de satisfacción corporal favorecen el desarrollo de una autoestima positiva. Por lo que habría que encaminar el proceso de enseñanza hacia este fin, así, por ejemplo, si el alumnado reconoce sus capacidades y limitaciones, las implicaciones en su autoestima, serian escasas o nulas (Infante et al., 2011).
La imagen corporal como concepto dinámico en la preadolescencia, necesita de un programa de educación física concretado, que tenga en cuenta el desarrollo de la competencia crítica con su entorno: la influencia de los medios de comunicación, las tendencias de los adolescentes y adultos. También, se estima necesario el desarrollo de un trabajo actitudinal y la incorporación de las familias al proceso de enseñanza, con el fin de contribuir al desarrollo integral como seres humanos. De esta manera, partiendo de los factores reales que influyen en el desarrollo del alumnado, se sientan las bases para diseñar e implementar un programa de intervención integral en educación física, específico para toda la zona educativa. Con ello, como la literatura científica nos muestra, se puede contribuir a la disminución del abandono escolar (Comisión Europea, 2014), la mejora de las calificaciones y contribuir a disminuir las desigualdades sociales que emergen en España, como señala el informe PISA (2015).

Este estudio se ha limitado a una zona sociocultural concreta, buscando la mejora del proceso de enseñanza a través del conocimiento singular de los factores influyentes en un medio sociocultural específico. Serviría como base para otras zonas, pero teniendo en cuenta la idiosincrasia del lugar.

También hay que señalar que han aparecido suficientes indicios de influencias externas que hacen que la actitud del alumnado hacia su cuerpo y la relación con los demás esté cambiando, y que se necesitarían investigaciones específicas, interdisciplinares y profundas, que contribuyeran a comprender el impacto de estos fenómenos en nuevas poblaciones.

\section{Referencias}

Arkin, H., \& Colton, R. (1984). An outline of statistical methods as applied to economics, business, education, social and physical science. New York: Barnes \& Noble.

Baile, J.I., Raich, R., \&Garrido, E. (2003). Evaluación de la insatisfacción corporal en adolescentes: efecto de administración 
de una escala. Anales de psicología, 10(2), 187-192.

Baldwin, S.A., \& Hoffmann, J.P. (2002). The dynamics of self-esteem: A growth-curve analysis. Journal of Youth and Adolescence, 31, 101-113.

Berenguera, A., Fernández de Sanmamed, M. J., Pons, M., Pujol, E., Rodríguez, D., \&Saura, S. (2014). Escuchar, observar y comprender. Recuperando la narrativa en las Ciencias de la Salud. Aportaciones de la investigación cualitativa. Barcelona: InstitutUniversitarid'Investigació en AtencióPrimària Jordi Gol (IDIAP J. Gol).

Berk, L. E. (2007). Development through the lifespan (4th ed.). Boston, MA: Pearson Education.

Bryk, A., Harding, H., \& Greenberg, S. (2012). Contextual influences on inquiries into effective teaching and their implications for improving student learning. Harvard Educational Review, 82(1), 83-106. Recuperado de http://www. hepg.org/her/abstract/1192

Collins, M.E. (1991). Body figure perceptions and preferences amongpreadolescent children. International Journal of Eating Disorders, 10,199-208.

ComisiónEuropea/EACEA/Eurydice/Cedefop. (2014). La lucha contra el abandono temprano de la educación y la formación en Europa: estrategias, políticas y medidas. Informe de Eurydice y Cedefop. Luxemburgo: Oficina de publicaciones de la Unión Europea.

Consejería de Educación de la Junta de Andalucía (CEJA). (2012). Decreto 56/2012, de 6 de marzo, por el que se regulan las Zonas Educativas de Andalucía, las Redes Educativas, de aprendizaje permanente y de mediación y la organización y el funcionamiento de los Consejos de Coordinación de Zona.

Cumming, S.P., Standage, M., Loney, T., Gammon, C., Neville, H., Sherar, L.B., \&Malina,R.M. (2011). The mediating role of physical self-concept on relations between biologicalmaturity status and physical activity in adolescent females. Journal of Adolescence, 34, 465-473. http:// doi.org/10.1016/j.adolescence.2010.06.006

Davison, T. E., \& McCabe, M. P. (2006). Adolescent body image and psychosocial functioning. The Journal of social psychology, 146(1), 15-30.

Dietz, W.H., \& Robinson, T. (2005). Clinical Practice. Overweight in children and adolescents. N Engl J Med, 352(20), 2100-2109.

Dowson, J., \&Hendersen, L. (2001). The validity of a short version of the Body Shape Questionnaire. Psychiatry Research, 102, 263-271.

Dunn, J., Lewis,V., \& Patrick, S.(2010).The idealization of thin figures and appearance concerns in middle schoolchildren. Journal of Applied Biobehavioral Research, 15, 134-143.

Espinoza, L., Rodríguez, F., Gálvez, J., Vargas, P., \&Yáñez, R. (2011). Valoración del autoconcepto físico en estudiantes universitarios y su relación con la práctica deportiva. Revista Motricidad Humana 8(12), 22-26.

Fuentes, E. (2011). Adquisición y mantenimiento de hábitos de vida saludables en los escolares de primer ciclo de Educación Secundaria Obligatoria de Jerez de la Frontera (Cádiz). Tesis Doctoral: Universidad de Granada. Recuperado de http://digibug.ugr.es/bitstre am/10481/19435/1/20015690.pdf

Goñi, A., Ruiz De Azúa, S., \& Rodríguez, A. (2006). Manual del Cuestionario de Autoconcepto Físico (CAF). Manual. Madrid: EOS.

Grogan, S. (1999). Body image: understanding body dissatisfaction in men, women and children. London: Routledge.

Hammersley, M., \& Atkinson, P. (1995). Ethnography: Practices and principles. New York: Routledge. Retrieved December, 2, 2008.

Infante, G., Goñi, A.,\& Villarroel, J.D. (2011) Actividad física y autoconcepto, físico ygeneral, a lo largo de la edad adulta. Revista de Psicologia del Deporte, 20(2), 429-444. 
Johnson, R. B., \& Christensen, L. B. (2014). Educational research: quantitative, qualitative, and mixed approaches (5th ed.). Los Angeles, CA: Sage.

Käll, L. B., Nilsson, M., \&Lindén, T. (2014). The impact of a physical activity intervention program on academic achievement in a Swedish elementary school setting. Journal of school health, 84(8), 473-480.

Klomsten, A.T., Skaalvik, E.M., \&Espnes, G.A. (2004). Physical Self-Concept and Sports:Do Gender Differences Still Exist? Sex roles, 50(1), 119-127.

Kriemler, S., Meyer, U., Martin, E., van Sluijs, E., Andersen, L., \& Martin, B. (2011). Effect of school-based interventions on physical activity and fitness in children and adolescents: a review of reviews and systematic update. British Journal of Sports Medicine, 45(11), http://doi.org/10.1136/bjs ports-2011-090186

Latorre, A., del Rincón, D., \& Arnal, J. (2003). Bases Metodológicas de la Investigación Educativa. Barcelona: Ediciones Experiencia, S.L.

Liu, M., Wu, L., \& Ming, Q. (2015). How Does Physical Activity Intervention Improve Self-Esteem and Self-Concept in Children and Adolescents? Evidence from a MetaAnalysis. PLoS ONE, 10(8), e0134804. htt p://doi.org/10.1371/journal.pone.0134804.

Marsh, H. W., \& Martin, A. J. (2011). Academic self-concept and academic achievement: Relations and causal ordering. British Journal of Educational Psychology, 81(1), 59-77.

Marsh H.W., \& O'Mara, A.J. (2008). Selfconcept is as multidisciplinary as it is multidimensional. A review of theory, measurement, and practice in self-concept research. In: Marsh H.W, Craven RG and McInerney D.M. (eds) Self-Processes, Learning, and Enabling Human Potential: Dynamic New Approaches. Charlotte, NC: Information Age Press, pp. 87-115.

Mellor, D., Fuller-Tyszkiewicz, M., McCabe, M. P., Ricciardelli, L. A., Skouteris, H., \&Mussap, A. J. (2014). A test of the resource security and the body mass index reference point hypotheses of body dissatisfaction amongst adolescents in eight countries. Ethnicity Ehealth, 19(5), 548-564.

Miles, M.B. \& Huberman, A.M. (1994). Qualitative data analysis: An expanded sourcebook (2a ed.). Thousand Oaks, CA: Sage.

Morgan, P.J., Saunders, K.L., Lubans, D.R. (2012). Improving physical self-perception in adolescent boys from disadvantaged schools: psychological outcomes from the physical activity leaders randomized controlled trial. PediatrObes; 7:e27-e32.

Onís, M., \&Blössner, M. (2000). Prevalence and trends of overweight among preschool children in developing countries. Am J ClinNutr 72, 1032-1039.

Pisa, O. E. C. D. (2015). Draft Science Framework. 2014-07-17. Recuperado de http://www.oecd.org/pisa/pisaproducts/ DraftPISA2015ScienceFramework.pdf.

Ramos, M.M., Catena, A. \& Trujillo, H.M. (2004). Manual de métodos y técnicas de investigación en ciencias del comportamiento. Madrid: Biblioteca Nueva.

Rees, R., Caird, J., Dickson, K., Vigurs, C., \& Thomas J. (2013) The views of young people in the UK about obesity, body size, shape andweight: a systematic review. London: EPPI-Centre, Social Science Research Unit, Institute of Education, University of London.

Rosenberg, M. (1965). Society and the adolescent self-image. Princeton, NJ: Princeton University Press.

Schmidt, M., Valkanover, S., Roebers, C., \&Conzelmann, A. (2013). Promoting a functional physical self-concept in physical education: Evaluation of a 10-week intervention. European Physical Education Review, 19(2), 232-255.

Standage, M., Gillison, F. B., Ntoumanis, N., \&Treasure, D. C. (2012). Predicting students' physical activity and healthrelated well-being: A prospective crossdomain investigation of motivation across school physical education and exercise 
settings. Journal of Sport $\mathcal{E}$ Exercise Psychology, 2012(34), 37-60.

Thompson, J.K. (2004). Handbook of Eating Disorders and Obesity. New York: Wiley.

Thompson M.A., \& Gray J.J. (1995). Development and validation of a new bodyimage assessment scale. J PersAssess. 4(2), 258-69.

Taylor, S.J., \&Bogdan, R. (1987). Introducción a los métodos cualitativos. Barcelona:Paidós.

Xu, X., Mellor, D., Kiehne, M., Ricciardelli, L. A., McCabe, M. P., \&Xu, Y. (2010). Body dissatisfaction, engagement in body change behaviors and sociocultural influences on body image among Chinese adolescents. Bodyimage, 7(2), 156-164.

\section{Notas}

* Artículo de investigación. 